\title{
Keeping a Spacecraft on the Sun-Earth Line ${ }^{1}$
}

\author{
Carlos M. Roithmayr ${ }^{2}$ and Linda Kay-Bunnell ${ }^{3}$
}

\begin{abstract}
Measurements of Earth's atmosphere as it occults sunlight can be obtained advantageously from a spacecraft placed in the proximity of the Sun-Earth Lagrange point $\mathcal{L}_{2}$. Maintaining the condition of continuous solar occultation by all parts of the atmospheric disk requires that the displacement of the spacecraft perpendicular to the Sun-Earth line remains less than $200 \mathrm{~km}$. However, the gravitational force exerted by the Earth's moon must be negated by propulsion in order to meet this rather tight constraint. We provide an estimate of propulsive force needed to keep the spacecraft coincident with $\mathcal{L}_{2}$, as well as estimates of velocity increments needed to maintain various trajectories in the close vicinity of $\mathcal{L}_{2}$.
\end{abstract}

\section{Introduction}

The benefits of placing spacecraft in halo orbits about Lagrange or libration points are widely discussed in the literature, as are methods for controlling such orbits. Use of the collinear SunEarth Lagrange points in particular for the conduct of Earth and space science missions is reviewed, for example, in Refs. [1], [2], and [3]. Halo orbits, and the more general Lissajous orbits, typically involve displacements from the Sun-Earth line ranging from thousands to hundreds of thousands of kilometers. Such relatively large excursions can not be tolerated for certain missions proposed

\footnotetext{
${ }^{1}$ A previous version of this work was presented as Paper AAS 04-246 at the AAS/AIAA Space Flight Mechanics Meeting, Maui, Hawai i, February, 2004.

${ }^{2}$ Spacecraft and Sensors Branch, NASA Langley Research Center, Hampton, VA 23681-2199.

${ }^{3}$ Spacecraft and Sensors Branch, NASA Langley Research Center; currently with NASA Goddard Space Flight Center, Greenbelt, MD 20771.
} 
recently. For instance, as discussed in Ref. [3] it is advantageous to study Earth's atmosphere from the trans-Earth equilibrium point $\mathcal{L}_{2}$ via solar occultation in the near infrared spectra. From this unique vantage point the entire limb of the Earth provides permanent occultation, making it possible to obtain hourly measurements at all latitudes of the atmosphere as the Earth rotates and produce nearly global high-resolution three-dimensional maps of the geographic distribution of major atmospheric constituent gas species such as $\mathrm{CO}_{2}, \mathrm{O}_{3}, \mathrm{O}_{2}, \mathrm{CH}_{4}, \mathrm{H}_{2} \mathrm{O}$, and $\mathrm{N}_{2} \mathrm{O}$. Such measurements can not be obtained by spacecraft in low Earth orbit and have never before been achieved.

Maintaining the condition of continuous solar occultation by all parts of the atmospheric disk requires that the displacement of the spacecraft perpendicular to the Sun-Earth line remains less than $200 \mathrm{~km}$. Views of Earth occulting the Sun from various positions in the neighborhood of $\mathcal{L}_{2}$ are contained in Fig. 1. The rectangular border around each image marks a $1^{\circ} \times 0.65^{\circ}$ field of view. Figures 1a, b, and c show Earth centered in front of the solar disk when viewed from three points on the Sun-Earth line; the distance is $1.5082 \times 10^{6} \mathrm{~km}$ from Earth in Fig. 1a, 50,000 km farther away in Fig. 1b, and 50,000 km closer in Fig. 1c. In Figs. 1d and e the vantage point is 1.5082 $\times 10^{6} \mathrm{~km}$ from Earth along the Sun-Earth line, and displaced in the ecliptic plane by some distance in a direction perpendicular to the Sun-Earth line. From a perpendicular displacement of $200 \mathrm{~km}$ the Earth is somewhat off center but still completely within the solar disk as displayed in Fig. 1d; however, a majority of Earth's limb fails to occult the Sun from a perpendicular displacement of $5,000 \mathrm{~km}$ as one can see in Fig. 1e.

As is well known from the study of the circular restricted three-body problem, a particle at $\mathcal{L}_{2}$ is considered to be in dynamic equilibrium based on the assumptions that the Sun and Earth orbit their common barycenter circularly and, more importantly, that only these two bodies exert gravitational force on the particle. The gravitational attraction of the Earth's moon (a fourth body) displaces the point of equilibrium by nearly $5,000 \mathrm{~km}$ in a roughly circular path with the period of the lunar cycle; although a spacecraft excursion of this amount in a direction parallel to the Sun-Earth line would not violate the science requirement, displacement of this magnitude in the perpendicular direction is unacceptable. Solutions of the circular restricted three-body problem reveal the existence of nearly rectilinear orbits in the halo family as described in Refs. [4] and [5]; however, the resulting displacement is perpendicular to the line between the primaries, and the 


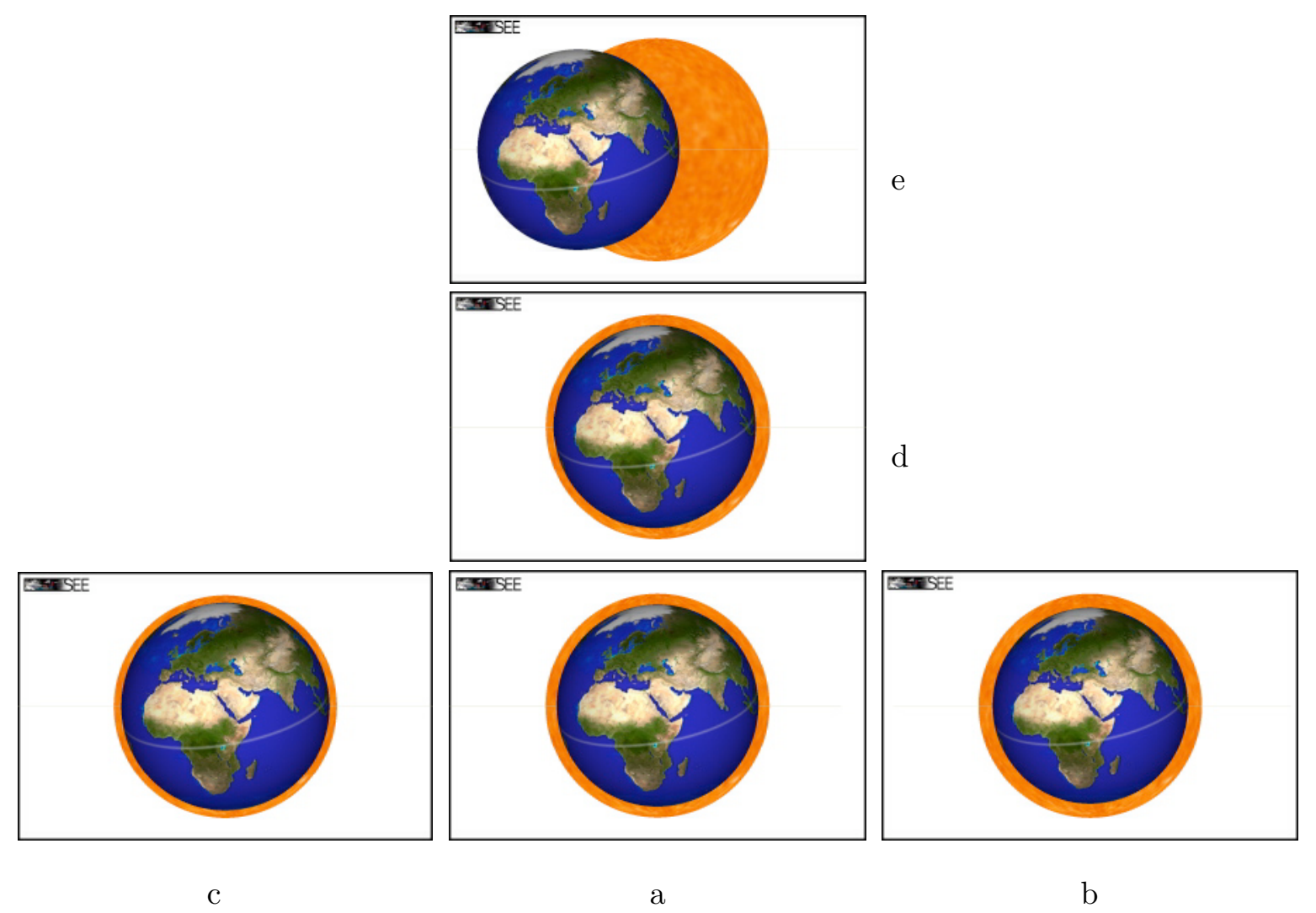

Figure 1: Views from the neighborhood of $\mathcal{L}_{2}$ 
perturbation exerted by a fourth body is not accounted for in this result. Consequently, a spacecraft such as the one proposed in Ref. [3] must possess a propulsion system capable of offsetting the lunar gravitational attraction; preliminary design of the system requires an estimate of the associated force per unit mass and corresponding velocity increment. Previous research, reported in Ref. [6], has determined that in the restricted four-body problem the position of a dynamic equilbrium lies on a line joining the Sun and the Earth-Moon barycenter. Motion in the vicinity of this equilibrium has been analyzed in that work as well. In this paper, however, we are concerned with motion near Sun-Earth $\mathcal{L}_{2}$, a point that ceases to correspond to equilibrium once the lunar gravitational perturbation is considered.

The paper proceeds as follows. First, we briefly review the approach taken by other researchers in showing that, to first order, one can neglect the solar gravitational perturbation at the collinear Earth-Moon libration points. This approach is then applied to the collinear Sun-Earth equilibrium point $\mathcal{L}_{2}$ to obtain the first-order lunar perturbation, which does not vanish. After neglecting the inclination of the lunar orbit plane relative to the ecliptic we obtain approximate expressions for projections of the lunar perturbation in directions parallel and perpendicular to the Sun-Earth line; these are in turn used to show the approximate displacement of the equilibrium point from $\mathcal{L}_{2}$. It becomes clear right away that a spacecraft coincident with the perturbed equilibrium will be displaced well in excess of $200 \mathrm{~km}$ from the Sun-Earth line. An exact expression for the propulsive force needed to counter the lunar gravity is evaluated numerically over one lunar cycle using positions of the Earth, Sun, and Moon specified by published ephemerides; approximate expressions for the two ecliptic-plane projections are seen to agree well with the exact results. Analytic integration of the relationships for the two projections furnishes an estimate of the velocity increment $\Delta V$ expected from a propulsion system for one lunar cycle in the event the spacecraft is to remain coincident with $\mathcal{L}_{2}$. Velocity increments needed to keep the spacecraft fixed at other points on the Sun-Earth line are also provided. Fixing the spacecraft is admittedly overly restrictive; as mentioned previously, solar occultation by the atmosphere will occur even when the spacecraft is permitted to move a reasonable distance along the Sun-Earth line. Thus, less propellant is needed if thrusters are used solely to offset the lunar perturbing force perpendicular to the Sun-Earth line. We conclude by quantifying reductions in the required velocity increments made possible by some trajectories that stay within the immediate neighborhood of $\mathcal{L}_{2}$. 


\section{Perturbation of Collinear Libration Points}

Previous researchers have addressed the solar gravitational perturbation of spacecraft motion near the collinear Earth-Moon libration points. It is instructive to review briefly their approach before employing it to study the lunar perturbation of a collinear Sun-Earth libration point. Colombo was the first to investigate the matter. In Ref. [7] he shows that, for a particle coincident with

a libration point, the solar perturbation can be neglected to first order. In Ref. [8], Nicholson carries the analysis to second order and applies it to motion within $10 \mathrm{~km}$ of the cislunar point $\mathcal{L}_{1}$. Farquhar treats the solar perturbation of an object in a periodic orbit about a collinear point in Ref. [9].

Figure 2 depicts a system of four particles $P_{i}$, each of mass $m_{i}(i=1,2,3,4)$, moving in a Newtonian reference frame $N$ under the influence of mutual gravitational attraction. We are particularly interested in a system where $P_{1}, P_{2}, P_{3}$, and $P_{4}$ represent respectively the Earth, a spacecraft, the Sun, and the Moon. Using the notation employed in Ref. [10], r indicates the position vector from $P_{1}$ to $P_{2}$. The vectors $\mathbf{d}_{i}$ represent the position vectors from $P_{i}$ to $P_{2}(i=3,4)$ and are used to express the direct effects of these particles on $P_{2}$, whereas the indirect effects are expressed in terms of the position vectors $\boldsymbol{\rho}_{i}$ from $P_{1}$ to $P_{i}(i=3,4)$.

\section{Solar Perturbation of Collinear Earth-Moon Libration Points}

Nicholson's demonstration makes use of two equations of motion relative to Earth, one for the spacecraft and one for the Moon, and a third expression relating the libration point position to the position of the Moon. The motion of a spacecraft relative to Earth, perturbed by the Sun and Moon, can be described with the aid of Eq. (8.55) in Ref. [10] where the number of particles $n$ is 4 .

$$
\frac{{ }^{N} d^{2}}{d t^{2}} \mathbf{r}+\frac{G\left(m_{1}+m_{2}\right) \mathbf{r}}{r^{3}}=-G\left[m_{3}\left(\frac{\mathbf{d}_{3}}{d_{3}{ }^{3}}+\frac{\boldsymbol{\rho}_{3}}{\rho_{3}{ }^{3}}\right)+m_{4}\left(\frac{\mathbf{d}_{4}}{d_{4}{ }^{3}}+\frac{\boldsymbol{\rho}_{4}}{\rho_{4}{ }^{3}}\right)\right]
$$

(Nicholson immediately neglects $G m_{2}$ in comparison to $G m_{1}$.) The motion of the Moon relative to Earth, perturbed by the Sun, is governed by the vector differential equation

$$
{ }^{N} \frac{d^{2}}{d t^{2}} \boldsymbol{\rho}_{4}+G\left(m_{1}+m_{4}\right) \frac{\boldsymbol{\rho}_{4}}{\rho_{4}{ }^{3}}=-G m_{3}\left(-\frac{\mathbf{z}}{z^{3}}+\frac{\boldsymbol{\rho}_{3}}{\rho_{3}{ }^{3}}\right)
$$

where $z$ is the magnitude of the position vector $\mathbf{z}$ from $P_{4}$, the Moon, to $P_{3}$, the Sun, which can be written as $\mathbf{z}=\boldsymbol{\rho}_{3}-\boldsymbol{\rho}_{4}$. 


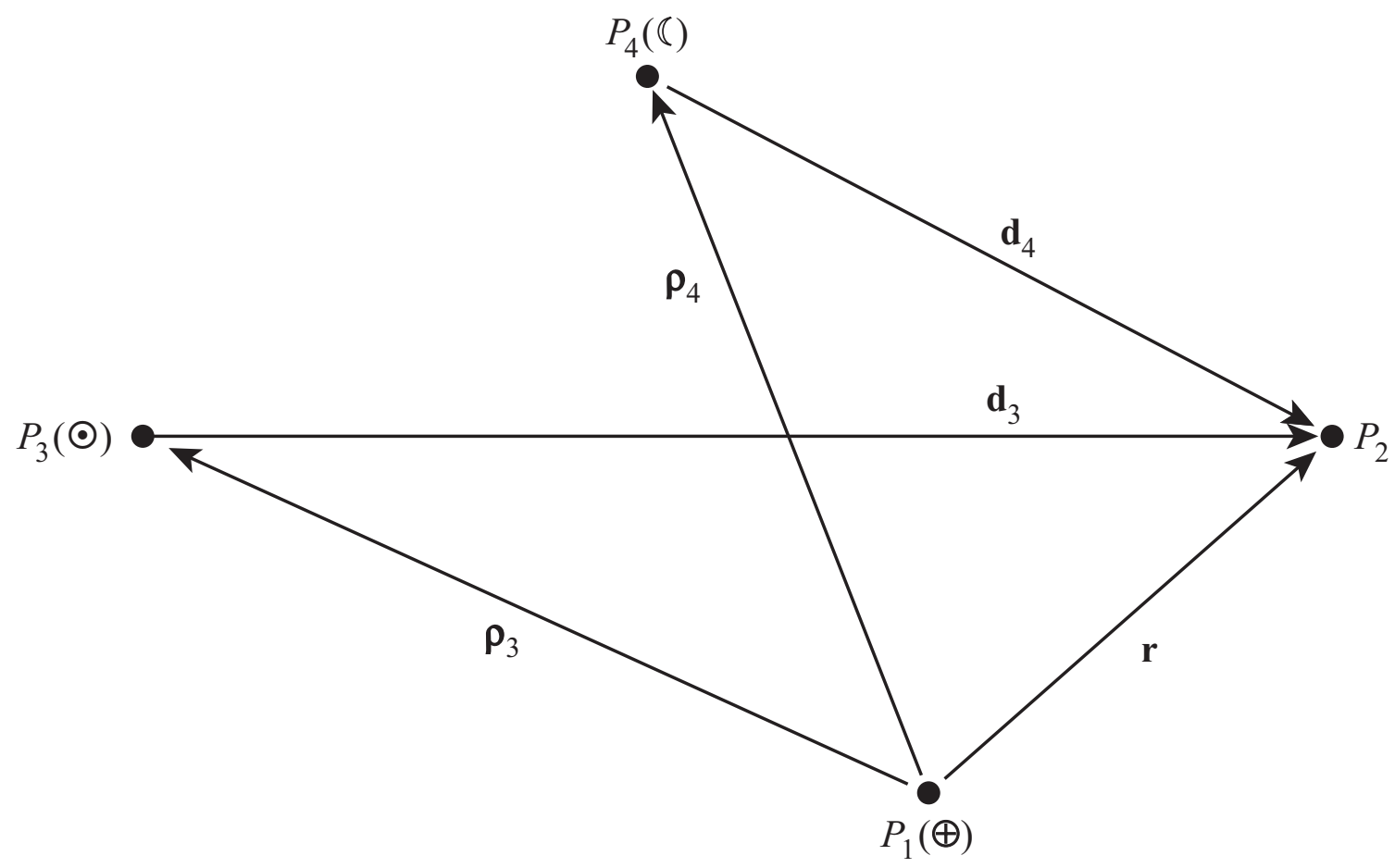

Figure 2: Four-Body System

In each relationship the solar term is expanded in a binomial series to obtain, for $r<\rho_{3}$ in the first instance,

$$
-G m_{3}\left(\frac{\mathbf{d}_{3}}{d_{3}{ }^{3}}+\frac{\boldsymbol{\rho}_{3}}{\rho_{3}{ }^{3}}\right)=-G m_{3}\left(\frac{\mathbf{r}-\boldsymbol{\rho}_{3}}{\left|\mathbf{r}-\boldsymbol{\rho}_{3}\right|^{3}}+\frac{\boldsymbol{\rho}_{3}}{\rho_{3}{ }^{3}}\right)=\frac{G m_{3}}{\rho_{3}{ }^{3}}\left(3 \hat{\boldsymbol{\rho}}_{3} \hat{\boldsymbol{\rho}}_{3}-\underline{\mathbf{U}}\right) \cdot \mathbf{r}+\cdots
$$

where $\hat{\boldsymbol{\rho}}_{3}$ is a unit vector having the same direction as $\boldsymbol{\rho}_{3}, \underline{\mathbf{U}}$ denotes the unit dyadic, and where the remaining terms are of second or higher degree in $r / \rho_{3}$. The right hand member of Eq. (2) is likewise expanded to obtain, for $\rho_{4}<\rho_{3}$,

$$
-G m_{3}\left(-\frac{\mathbf{z}}{z^{3}}+\frac{\boldsymbol{\rho}_{3}}{\rho_{3}{ }^{3}}\right)=-G m_{3}\left(\frac{\boldsymbol{\rho}_{4}-\boldsymbol{\rho}_{3}}{\left|\boldsymbol{\rho}_{4}-\boldsymbol{\rho}_{3}\right|^{3}}+\frac{\boldsymbol{\rho}_{3}}{\rho_{3}{ }^{3}}\right)=\frac{G m_{3}}{\rho_{3}{ }^{3}}\left(3 \hat{\boldsymbol{\rho}}_{3} \hat{\boldsymbol{\rho}}_{3}-\underline{\mathbf{U}}\right) \cdot \boldsymbol{\rho}_{4}+\cdots
$$

In the absence of solar gravitation, the position vectors from $P_{1}$ to the three libration points on the Earth-Moon line are given by $\gamma_{i} \boldsymbol{\rho}_{4}$, where $\gamma_{1}, \gamma_{2}$, and $\gamma_{3}$ are three distinct constants whose values are determined by the masses of the Moon and Earth. When the spacecraft is coincident with one of the collinear libration points, the position vector $\mathbf{r}$ is given by

$$
\mathbf{r}=\gamma_{i} \boldsymbol{\rho}_{4} \quad(i=1,2,3)
$$

Relative velocity and acceleration of the coincident spacecraft are obtained by differentiating these relationships with respect to time in $N$. Because the ratios of $\mathbf{r}$ to $\boldsymbol{\rho}_{4},{ }^{N} d \mathbf{r} / d t$ to ${ }^{N} d \boldsymbol{\rho}_{4} / d t$, and 
${ }^{N} d^{2} \mathbf{r} / d t^{2}$ to ${ }^{N} d^{2} \boldsymbol{\rho}_{4} / d t^{2}$ are all given by the same constant $\gamma_{i}$, Colombo calls the motion of a libration point homothetic to the motion of the Moon relative to the Earth. The relationship involving the second derivative plays a key role in what follows.

$$
\frac{{ }^{N} d^{2}}{d t^{2}} \mathbf{r}=\gamma_{i} \frac{{ }^{N}}{d t^{2}} \boldsymbol{\rho}_{4} \quad(i=1,2,3)
$$

The solar perturbation at the collinear Earth-Moon equilibrium points is shown to be negligible to first order by substituting from Eqs. (3) and (4) into (1) and (2) respectively, and substituting from the resulting expressions into (6).

$$
\begin{aligned}
{ }^{N} \frac{d^{2}}{d t^{2}} \mathbf{r}-\gamma_{i} \frac{{ }^{N}}{d t^{2}} \boldsymbol{\rho}_{4}= & -\frac{G m_{1} \mathbf{r}}{r^{3}}-G m_{4}\left(\frac{\mathbf{d}_{4}}{d_{4}{ }^{3}}+\frac{\boldsymbol{\rho}_{4}}{\rho_{4}{ }^{3}}\right)+\frac{G m_{3}}{\rho_{3}{ }^{3}}\left(3 \hat{\boldsymbol{\rho}}_{3} \hat{\boldsymbol{\rho}}_{3}-\underline{\mathbf{U}}\right) \cdot \mathbf{r}+\cdots \\
& +G\left(m_{1}+m_{4}\right) \gamma_{i} \frac{\boldsymbol{\rho}_{4}}{\rho_{4}{ }^{3}}-\frac{G m_{3}}{\rho_{3}{ }^{3}}\left(3 \hat{\boldsymbol{\rho}}_{3} \hat{\boldsymbol{\rho}}_{3}-\underline{\mathbf{U}}\right) \cdot \gamma_{i} \boldsymbol{\rho}_{4}-\cdots \quad(i=1,2,3)
\end{aligned}
$$

where $G m_{2}$ has been neglected in comparison to $G m_{1}$. Upon appealing to Eqs. (5), it can be seen immediately that the terms involving $\mathrm{Gm}_{3}$ cancel one another. If the surviving terms on the right hand side are to vanish, thereby satisfying Eqs. (6), there arise polynomials that are quintic in $\gamma_{i}$ with coefficients that are related to the ratio of the masses of the Moon and Earth.

\section{Lunar Perturbation of Collinear Sun-Earth Libration Point $\mathcal{L}_{2}$}

An approach similar to the one taken by Colombo and Nicholson can be followed to analyze the lunar perturbation at the collinear Sun-Earth libration point $\mathcal{L}_{2}$. In contrast to their results for the Earth-Moon system, the lunar perturbation to the Sun-Earth system does not vanish, even to first order.

We begin with a counterpart to Eq. (1),

$$
\frac{{ }^{N}}{d t^{2}} \mathbf{r}+\frac{G\left(m_{1}+m_{2}\right) \mathbf{r}}{r^{3}}=\frac{\mathbf{p}}{m_{2}}-G\left[m_{3}\left(\frac{\mathbf{d}_{3}}{d_{3}{ }^{3}}+\frac{\boldsymbol{\rho}_{3}}{\rho_{3}{ }^{3}}\right)+m_{4}\left(\frac{\mathbf{d}_{4}}{d_{4}{ }^{3}}+\frac{\boldsymbol{\rho}_{4}}{\rho_{4}{ }^{3}}\right)\right]
$$

where $\mathbf{p} / m_{2}$ is a propulsive force per unit mass that can be applied to $P_{2}$ to control its motion. In place of Eq. (2) we use an equation governing the motion of the Sun relative to the Earth, perturbed by the Moon.

$$
\frac{{ }^{N}}{d t^{2}} \boldsymbol{\rho}_{3}+G\left(m_{1}+m_{3}\right) \frac{\boldsymbol{\rho}_{3}}{\rho_{3}{ }^{3}}=-G m_{4}\left(\frac{\mathbf{z}}{z^{3}}+\frac{\boldsymbol{\rho}_{4}}{\rho_{4}{ }^{3}}\right)
$$


The lunar term in Eq. (8) is expanded in a binomial series as follows. After recognizing that $\boldsymbol{\rho}_{4}+\mathbf{d}_{4}=\mathbf{r}$, one may write

$$
-G m_{4}\left(\frac{\mathbf{d}_{4}}{d_{4}{ }^{3}}+\frac{\boldsymbol{\rho}_{4}}{\rho_{4}{ }^{3}}\right)=-G m_{4}\left(\frac{\mathbf{r}-\boldsymbol{\rho}_{4}}{\left|\mathbf{r}-\boldsymbol{\rho}_{4}\right|^{3}}+\frac{\boldsymbol{\rho}_{4}}{\rho_{4}{ }^{3}}\right)
$$

The distance $r$ from Earth to $\mathcal{L}_{2}$ is approximately $1.5 \times 10^{6} \mathrm{~km}$, or four times the distance $\rho_{4}$ from Earth to the Moon, 384,400 km. Thus, we factor out $r$ from the denominator of the first term

$$
\frac{1}{\left|\mathbf{r}-\boldsymbol{\rho}_{4}\right|^{3}}=\left[\left(\mathbf{r}-\boldsymbol{\rho}_{4}\right) \cdot\left(\mathbf{r}-\boldsymbol{\rho}_{4}\right)\right]^{-\frac{3}{2}}=r^{-3}\left[1-2 \frac{\mathbf{r} \cdot \boldsymbol{\rho}_{4}}{r^{2}}+\left(\frac{\rho_{4}}{r}\right)^{2}\right]^{-\frac{3}{2}}
$$

and define $x$ as

$$
x \triangleq-2 \frac{\mathbf{r} \cdot \boldsymbol{\rho}_{4}}{r^{2}}+\left(\frac{\rho_{4}}{r}\right)^{2}
$$

Neglecting the inclination of the Moon's orbit plane to the ecliptic, about $5^{\circ}$, the largest value of $x$ is found to be about 0.58 , whereas the smallest value is approximately -0.45 ; hence, $-1<x<1$, and one can employ the binomial series

$$
(1+x)^{-\frac{3}{2}}=1-\frac{3}{2} x+\frac{15}{8} x^{2}-\cdots
$$

to write

$$
\left[1-2 \frac{\mathbf{r} \cdot \boldsymbol{\rho}_{4}}{r^{2}}+\left(\frac{\rho_{4}}{r}\right)^{2}\right]^{-\frac{3}{2}}=1+3 \frac{\mathbf{r} \cdot \boldsymbol{\rho}_{4}}{r^{2}}+\cdots
$$

where the remaining terms are of second or higher degree in $\rho_{4} / r$. Substitution from Eq. (14) into (11) and then into (10) yields, for $r>\rho_{4}$,

$$
\begin{aligned}
-G m_{4}\left(\frac{\mathbf{d}_{4}}{d_{4}{ }^{3}}+\frac{\boldsymbol{\rho}_{4}}{\rho_{4}{ }^{3}}\right) & =-G m_{4}\left[\frac{\mathbf{r}-\boldsymbol{\rho}_{4}}{r^{3}}\left(1+3 \frac{\mathbf{r} \cdot \boldsymbol{\rho}_{4}}{r^{2}}+\cdots\right)+\frac{\boldsymbol{\rho}_{4}}{\rho_{4}{ }^{3}}\right] \\
& =-G m_{4}\left\{\left[\frac{3 \hat{\mathbf{r}} \hat{\mathbf{r}}}{r^{3}}+\left(\frac{1}{\rho_{4}{ }^{3}}-\frac{1}{r^{3}}\right) \underline{\mathbf{U}}\right] \cdot \boldsymbol{\rho}_{4}+\frac{\mathbf{r}}{r^{3}}+\cdots\right\}
\end{aligned}
$$

where $\hat{\mathbf{r}}$ is a unit vector having the same direction as $\mathbf{r}$, and a term involving $\left(\rho_{4} / r\right)^{2}$ has been neglected in the final step. Proceeding similarly with the lunar perturbation in Eq. (9), we obtain

$$
\begin{aligned}
-G m_{4}\left(\frac{\mathbf{z}}{z^{3}}+\frac{\boldsymbol{\rho}_{4}}{\rho_{4}{ }^{3}}\right) & =-G m_{4}\left(\frac{\boldsymbol{\rho}_{3}-\boldsymbol{\rho}_{4}}{\left|\boldsymbol{\rho}_{3}-\boldsymbol{\rho}_{4}\right|^{3}}+\frac{\boldsymbol{\rho}_{4}}{\rho_{4}{ }^{3}}\right) \\
& =-G m_{4}\left\{\left[\frac{3 \hat{\boldsymbol{\rho}}_{3} \hat{\boldsymbol{\rho}}_{3}}{\rho_{3}{ }^{3}}+\left(\frac{1}{\rho_{4}{ }^{3}}-\frac{1}{\rho_{3}{ }^{3}}\right) \underline{\mathbf{U}}\right] \cdot \boldsymbol{\rho}_{4}+\frac{\boldsymbol{\rho}_{3}}{\rho_{3}{ }^{3}}+\cdots\right\}
\end{aligned}
$$

for $\rho_{3}>\rho_{4}$. 
As counterparts to Eqs. (5) and (6) we require expressions involving the position of a spacecraft that is coincident with the trans-Earth libration point on the Sun-Earth line,

$$
\mathbf{r}=-\gamma_{2} \boldsymbol{\rho}_{3} \quad\left(0<\gamma_{2}<1\right)
$$

and

$$
\frac{{ }^{N}}{d t^{2}} \mathbf{r}=-\gamma_{2}{ }^{N} \frac{d^{2}}{d t^{2}} \boldsymbol{\rho}_{3}
$$

Substitution from Eqs. (8), (9), (15), and (16) into (18) yields

$$
\begin{aligned}
& { }^{N} \frac{d^{2}}{d t^{2}} \mathbf{r}+\gamma_{2} \frac{{ }^{N}}{d t^{2}} \boldsymbol{\rho}_{3}= \\
& \quad-\frac{G m_{1} \mathbf{r}}{r^{3}}+\frac{\mathbf{p}}{m_{2}}-G m_{3}\left(\frac{\mathbf{d}_{3}}{d_{3}{ }^{3}}+\frac{\boldsymbol{\rho}_{3}}{\rho_{3}{ }^{3}}\right)-G m_{4}\left\{\left[\frac{3 \hat{\mathbf{r}} \hat{\mathbf{r}}}{r^{3}}+\left(\frac{1}{\rho_{4}{ }^{3}}-\frac{1}{r^{3}}\right) \underline{\mathbf{U}}\right] \cdot \boldsymbol{\rho}_{4}+\frac{\mathbf{r}}{r^{3}}+\cdots\right\} \\
& \quad-G\left(m_{1}+m_{3}\right) \gamma_{2} \frac{\boldsymbol{\rho}_{3}}{\rho_{3}{ }^{3}}-G m_{4} \gamma_{2}\left\{\left[\frac{3 \hat{\boldsymbol{\rho}}_{3} \hat{\boldsymbol{\rho}}_{3}}{\rho_{3}{ }^{3}}+\left(\frac{1}{\rho_{4}{ }^{3}}-\frac{1}{\rho_{3}{ }^{3}}\right) \underline{\mathbf{U}}\right] \cdot \boldsymbol{\rho}_{4}+\frac{\boldsymbol{\rho}_{3}}{\rho_{3}{ }^{3}}+\cdots\right\}
\end{aligned}
$$

where $G m_{2}$ has been neglected in comparison to $G m_{1}$.

\section{Perturbed Motion in the Neighborhood of $\mathcal{L}_{2}$}

With suitable initial position and velocity, a spacecraft will remain coincident with $\mathcal{L}_{2}$ when the right hand member of Eq. (19) vanishes. The propulsive force per unit mass $\mathbf{p} / m_{2}$ can be used to counter the lunar gravitational perturbation as will be discussed presently. The terms involving $G m_{1}$ and $G m_{3}$ can be collected and set equal to zero, giving rise to a quintic polynomial for $\gamma_{2}$; the result given in Ref. [9] is $1.0037 \times 10^{-2}$.

The lunar perturbational force per unit mass acting on a spacecraft coincident with $\mathcal{L}_{2}$ is developed as follows. In view of Eq. (17) we observe that $\boldsymbol{\rho}_{3}=-\mathbf{r} / \gamma_{2}, \rho_{3}=r / \gamma_{2}$, and $\hat{\boldsymbol{\rho}}_{3}=-\hat{\mathbf{r}}$. Thus,

$$
\begin{aligned}
\frac{\mathbf{f}}{m_{2}} \triangleq & -G m_{4}\left\{\left[\frac{3 \hat{\mathbf{r}} \hat{\mathbf{r}}}{r^{3}}+\left(\frac{1}{\rho_{4}{ }^{3}}-\frac{1}{r^{3}}\right) \underline{\mathbf{U}}\right] \cdot \boldsymbol{\rho}_{4}+\frac{\mathbf{r}}{r^{3}}+\cdots\right\} \\
& -G m_{4} \gamma_{2}\left\{\left[\frac{3 \hat{\boldsymbol{\rho}}_{3} \hat{\boldsymbol{\rho}}_{3}}{\rho_{3}{ }^{3}}+\left(\frac{1}{\rho_{4}{ }^{3}}-\frac{1}{\rho_{3}{ }^{3}}\right) \underline{\mathbf{U}}\right] \cdot \boldsymbol{\rho}_{4}+\frac{\boldsymbol{\rho}_{3}}{\rho_{3}{ }^{3}}+\cdots\right\} \\
= & -G m_{4}\left\{\left[\frac{3 \hat{\mathbf{r}} \hat{\mathbf{r}}}{r^{3}}+\left(\frac{1}{\rho_{4}{ }^{3}}-\frac{1}{r^{3}}\right) \underline{\mathbf{U}}\right] \cdot \boldsymbol{\rho}_{4}+\frac{\mathbf{r}}{r^{3}}+\cdots\right\} \\
& -G m_{4} \gamma_{2}\left\{\left[\frac{3 \gamma_{2}^{3} \hat{\mathbf{r}} \hat{\mathbf{r}}}{r^{3}}+\left(\frac{1}{\rho_{4}^{3}}-\frac{\gamma_{2}{ }^{3}}{r^{3}}\right) \underline{\mathbf{U}}\right] \cdot \boldsymbol{\rho}_{4}-\frac{\mathbf{r}}{\gamma_{2}} \frac{\gamma_{2}{ }^{3}}{r^{3}}+\cdots\right\} \\
= & -G m_{4}\left\{\left[\frac{3 \hat{\mathbf{r}} \hat{\mathbf{r}}}{r^{3}}\left(1+\gamma_{2}{ }^{4}\right)+\left(\frac{1+\gamma_{2}}{\rho_{4}{ }^{3}}-\frac{1+\gamma_{2}}{r^{3}}\right) \underline{\mathbf{U}}\right] \cdot \boldsymbol{\rho}_{4}+\left(1-\gamma_{2}{ }^{3}\right) \frac{\mathbf{r}}{r^{3}}+\cdots\right\}
\end{aligned}
$$


It is worth noting that $1+\gamma_{2} \approx 1+10^{-2} \approx 1,1-\gamma_{2}{ }^{3} \approx 1-10^{-6} \approx 1$, and $1+\gamma_{2}{ }^{4} \approx 1+10^{-8} \approx 1$.

It is clear from Eq. (20) that the lunar perturbation can not be discarded from the right hand member of Eq. (19). In contrast, the solar perturbation disappears (to first order) from Eqs. (7). This observation can be accompanied by others regarding the relative magnitudes of the position vectors involved in the direct terms of the perturbations in Eqs. (15) and (16) in the first instance, and in Eqs. (3) and (4) in the second instance. In Eq. (3) the position vector $\mathbf{d}_{3}$ in the direct term is written as the difference $\mathbf{r}-\boldsymbol{\rho}_{3}$. As noted previously, $r<\rho_{3}$; thus, a denominator $1 / \rho_{3}{ }^{3}$ is factored out in the binomial expansion of the direct term and a cancellation of the indirect term $\boldsymbol{\rho}_{3} / \rho_{3}{ }^{3}$ occurs when the direct and indirect terms are added together. The position vector $\mathbf{z}=-\left(\boldsymbol{\rho}_{4}-\boldsymbol{\rho}_{3}\right)$ in the direct term in Eq. (4) is treated in an analagous manner when $\rho_{4}<\rho_{3}$. In constrast, the position vector $\mathbf{d}_{4}$ involved in the direct term in Eq. (15) is written as $\mathbf{r}-\boldsymbol{\rho}_{4}$ but in this case $r>\rho_{4}$ so $1 / r^{3}$ is factored out in the binomial expansion rather than $1 / \rho_{4}{ }^{3}$, and there is no cancellation of the indirect term $\boldsymbol{\rho}_{4} / \rho_{4}{ }^{3}$ when the sum of the direct and indirect terms is formed. A similar situation exists with $\mathbf{z}=\boldsymbol{\rho}_{3}-\boldsymbol{\rho}_{4}$ in the direct term in Eq. (16), with $\rho_{3}>\rho_{4}$.

Consider a reference frame $A$, shown in Fig. 3 , in which the line passing through $P_{3}, P_{1}$ and $\mathcal{L}_{2}$ is fixed. It is convenient to introduce three mutually perpendicular unit vectors $\hat{\mathbf{a}}_{1}, \hat{\mathbf{a}}_{2}$, and $\hat{\mathbf{a}}_{3}$ fixed in $A$, where $\hat{\mathbf{a}}_{1}=\hat{\mathbf{r}}$, $\hat{\mathbf{a}}_{2}$ lies in the ecliptic plane, and $\hat{\mathbf{a}}_{3}=\hat{\mathbf{a}}_{1} \times \hat{\mathbf{a}}_{2}$. In view of the negligible inclination of the Moon's orbit plane to the ecliptic, $\boldsymbol{\rho}_{4}$ can be approximated by

$$
\boldsymbol{\rho}_{4} \approx \rho_{4}\left(\cos \theta_{4} \hat{\mathbf{a}}_{1}+\sin \theta_{4} \hat{\mathbf{a}}_{2}\right)
$$

where $\rho_{4}$ is regarded as constant, and where the angle between $\mathbf{r}$ and $\boldsymbol{\rho}_{4}$ is given by $\theta_{4}=n_{s} t \triangleq$ $\left(n_{4}-n_{3}\right) t$. The mean motions of the Earth-Moon and Sun-Earth orbits are denoted by $n_{4}$ and

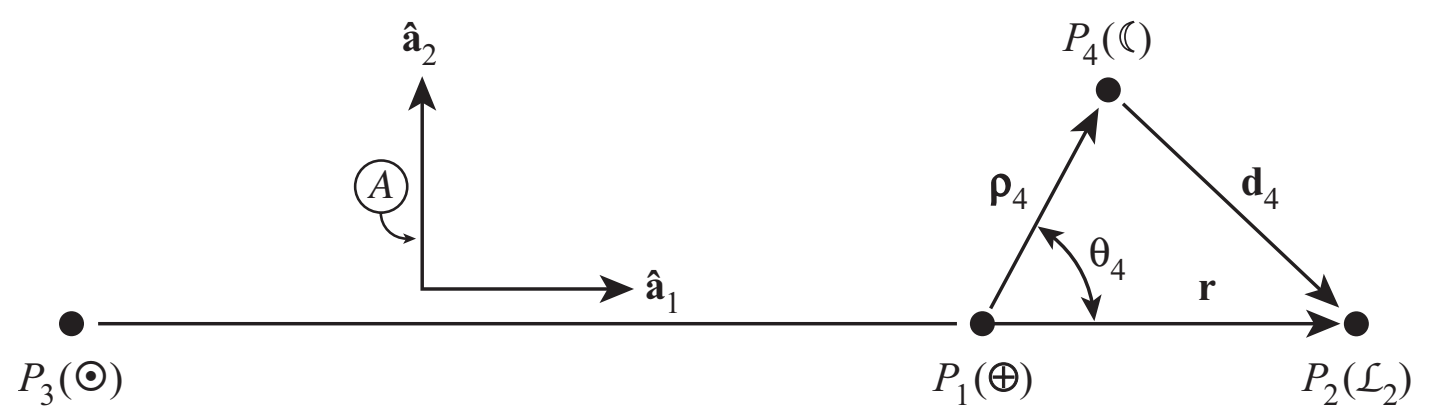

Figure 3: Spacecraft on the Sun-Earth Line 
$n_{3}$ respectively, and $\theta_{4}$ goes from 0 to $2 \pi$ during one lunar synodical period. Using the terms that appear in Eq. (20), projections of $\mathbf{f} / m_{2}$ in the directions of $\hat{\mathbf{a}}_{1}$ and $\hat{\mathbf{a}}_{2}$ are given by

$$
\begin{aligned}
& f_{1} \triangleq \frac{\mathbf{f} \cdot \hat{\mathbf{a}}_{1}}{m_{2}}=-G m_{4}\left\{\left[\frac{1+\gamma_{2}}{\rho_{4}{ }^{3}}+\frac{2\left(1+\gamma_{2}^{4}\right)}{r^{3}}\right] \rho_{4} \cos \theta_{4}+\frac{1-\gamma_{2}{ }^{3}}{r^{2}}\right\} \\
& f_{2} \triangleq \frac{\mathbf{f} \cdot \hat{\mathbf{a}}_{2}}{m_{2}}=-G m_{4}\left(\frac{1+\gamma_{2}}{\rho_{4}{ }^{3}}-\frac{1+\gamma_{2}^{4}}{r^{3}}\right) \rho_{4} \sin \theta_{4}
\end{aligned}
$$

Linearized equations governing motion in the neighborhood of a collinear libration point in the circular restricted three-body problem are presented, for example, in Refs. [6], [8], and [9]. The derivation of these equations rests on the assumption that two massive bodies orbit each other in a circle of constant radius, and a third body whose motion is of interest has mass that is insignificant in comparison. The homogeneous form of the equations (Ref. [9]) is based on the additional assumption that the only forces acting on the third body are those due to gravitational attraction from the two primary bodies. A nonhomogeneous form accounts for a perturbing force; for example, the gravitational attraction of a fourth body. In the case of Ref. [8], components of solar gravitation acting at Earth-Moon $\mathcal{L}_{1}$ or $\mathcal{L}_{2}$ serve as forcing functions for the in-plane equations, whereas lunar gravitation acting at Sun-Earth $\mathcal{L}_{2}$ perturbs the in-plane motion in Ref. [6]. Here, we employ $f_{1}$ and $f_{2}$ to approximate the lunar perturbation acting near Sun-Earth $\mathcal{L}_{2}$. In the absence of propulsion, linear differential equations describing motion in the ecliptic plane are written as

$$
\begin{aligned}
\ddot{x}-2 n_{3} \dot{y}-\left(1+2 B_{L}\right) n_{3}{ }^{2} x & =f_{1} \\
\ddot{y}+2 n_{3} \dot{x}+\left(B_{L}-1\right) n_{3}{ }^{2} y & =f_{2}
\end{aligned}
$$

where in-plane displacements of the spacecraft from $\mathcal{L}_{2}$ are assumed to be small, and are denoted by $x=\left(\mathbf{r}+\gamma_{2} \boldsymbol{\rho}_{3}\right) \cdot \hat{\mathbf{a}}_{1}$ and $y=\left(\mathbf{r}+\gamma_{2} \boldsymbol{\rho}_{3}\right) \cdot \hat{\mathbf{a}}_{2}$. The constant $B_{L}$ for Sun-Earth $\mathcal{L}_{2}$ is given by

$$
B_{L}=\frac{1-\mu}{\left(1+\gamma_{2}\right)^{3}}+\frac{\mu}{\gamma_{2}^{3}}
$$

where the mass ratio for the Earth and Sun is simply $\mu=m_{1} /\left(m_{3}+m_{1}\right)$. A numerical value of 3.9408 for $B_{L}$ is reported in Ref. [9].

Using the numerical values in Table 1 , one finds

$$
\begin{aligned}
& f_{1}=-259 \cos n_{s} t-16 \quad \mathrm{~km} / \mathrm{day}^{2} \\
& f_{2}=-246 \sin n_{s} t \quad \mathrm{~km} / \text { day }^{2}
\end{aligned}
$$


Table 1: ASTRONOMICAL PARAMETERS

\begin{tabular}{ll}
\hline \hline$\rho_{4}$ & $384,400 \mathrm{~km}$ \\
$r$ & $1.50151 \times 10^{6} \mathrm{~km}$ \\
$G m_{1}$ & $3.986 \times 10^{5} \mathrm{~km}^{3} / \mathrm{s}^{2}$ \\
$G m_{3}$ & $1.327 \times 10^{11} \mathrm{~km}^{3} / \mathrm{s}^{2}$ \\
$G m_{4}$ & $4.903 \times 10^{3} \mathrm{~km}^{3} / \mathrm{s}^{2}$ \\
$n_{3}$ & $0.0172 \mathrm{rad} /$ day \\
$n_{4}$ & $0.2300 \mathrm{rad} /$ day \\
$\gamma_{2}$ & $1.0037 \times 10^{-2}$ \\
$B_{L}$ & 3.9408 \\
\hline \hline
\end{tabular}

in which case a particular solution of Eqs. (24) and (25) is given by

$$
\begin{aligned}
& x=4666 \cos n_{s} t+6177 \quad \mathrm{~km} \\
& y=4770 \sin n_{s} t \quad \mathrm{~km}
\end{aligned}
$$

In a study of the restricted four-body problem presented in Ref. [6] (or Ref. [9], pp. 42-44), Farquhar determines the location of an equilibrium point $O^{\prime}$ displaced from $\mathcal{L}_{2}$ by the lunar perturbation. A line joining the Sun and $O^{\prime}$ passes through the Earth-Moon barycenter, as shown in Fig. 4. The path described by Eqs. (29) and (30), marked by the symbol $\times$ in Fig. 5, is seen to be similar to the trajectory of $O^{\prime}$, indicated with an O. Corresponding points on the two paths are approximatley $250 \mathrm{~km}$ from each other. Data points are one day apart and motion proceeds

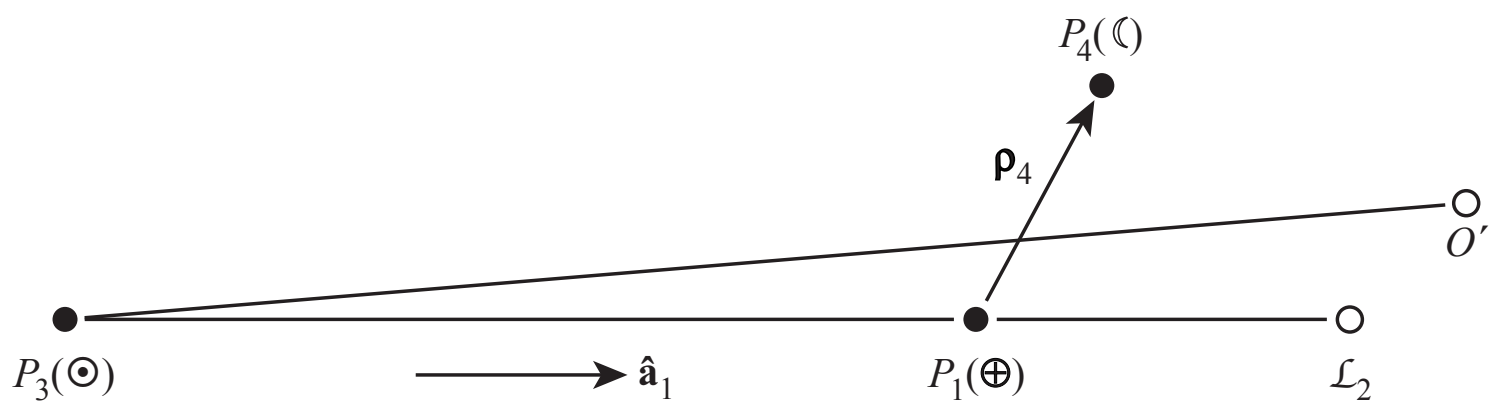

Figure 4: Equilibrium Point Perturbed by Lunar Gravitation 


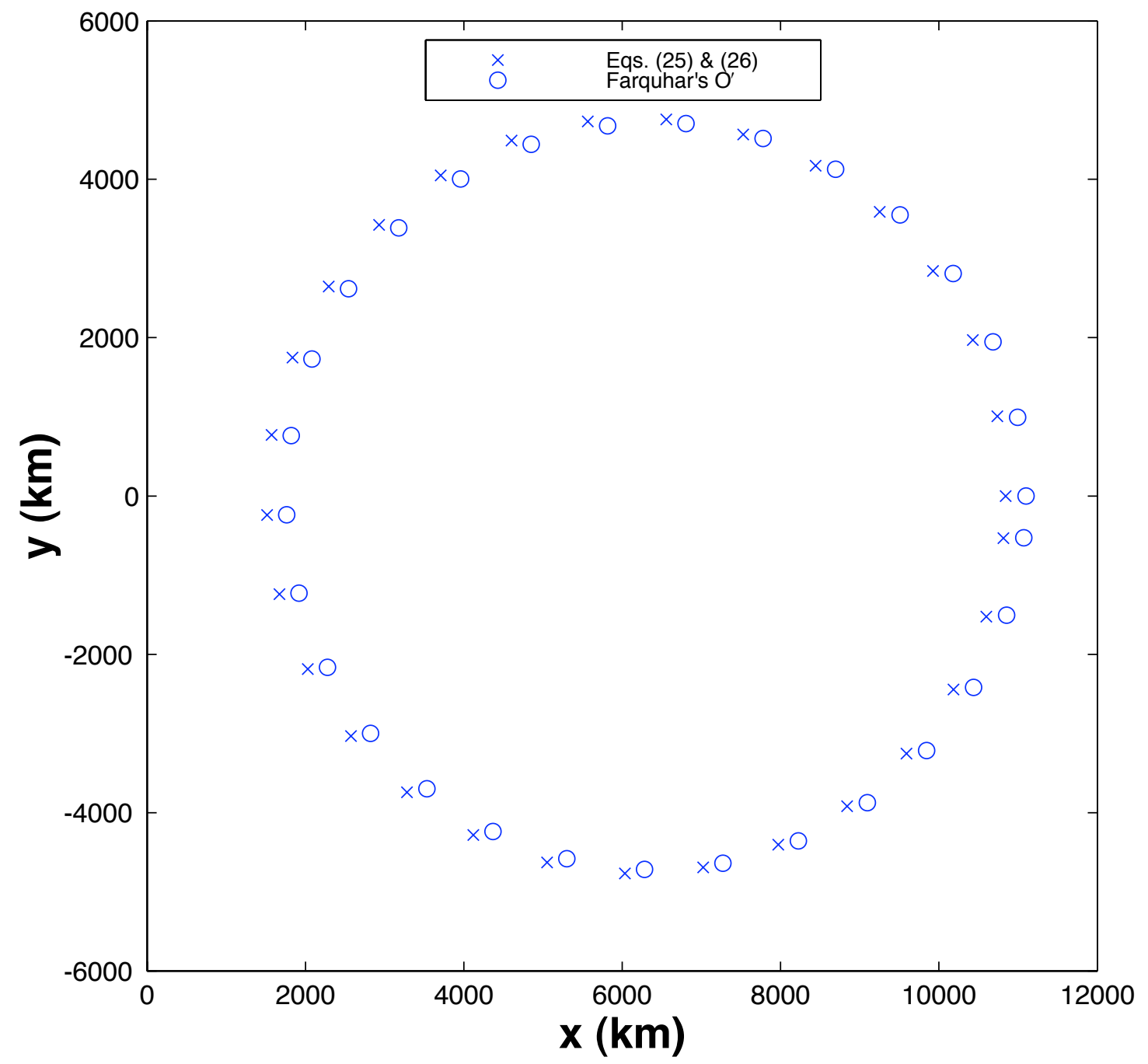

Figure 5: Perturbed Equilibrium Position Relative to Sun-Earth $\mathcal{L}_{2}$ 
counterclockwise. By accounting for higher order terms in the lunar perturbation Farquhar is able to show in Ref. [6] that a spacecraft remains within $20 \mathrm{~km}$ of $O^{\prime}$, as illustrated in Ref. [11]. Thus it would be possible, with proper initial conditions, for a spacecraft to move in the ecliptic plane unaided by propulsion such that it remains coincident with the equilibrium point in a $4666 \times 4770$ $\mathrm{km}$ ellipse centered on the Sun-Earth line about $6177 \mathrm{~km}$ farther away from the Earth than $\mathcal{L}_{2}$. However, this first order perturbed motion is unsatisfactory in the case of the Earth observation telescope for reasons discussed previously. The excursions in $y$ are not permissible even though the excursions in $x$ do not present a problem. Consequently, propulsion is required to negate the lunar perturbation at Sun-Earth $\mathcal{L}_{2}$.

\section{Propulsive Force Per Unit Mass}

A spacecraft can be made to remain coincident with $\mathcal{L}_{2}$ by countering the lunar perturbation with propulsion, in which case

$$
\mathbf{p} / m_{2}=-\mathbf{f} / m_{2}
$$

Referring to Eqs. (8), (9), and (18), an exact expression can be written as

$$
\frac{\mathbf{p}}{m_{2}}=G m_{4}\left[\left(\frac{\mathbf{d}_{4}}{d_{4}{ }^{3}}+\frac{\boldsymbol{\rho}_{4}}{\rho_{4}{ }^{3}}\right)+\gamma_{2}\left(\frac{\mathbf{z}}{z^{3}}+\frac{\boldsymbol{\rho}_{4}}{\rho_{4}{ }^{3}}\right)\right]
$$

Alternatively, the inclination of the Moon's orbit plane to the ecliptic can be neglected once again and, in view of Eqs. (22) and (23), an approximate relationship can be written

$$
\frac{\mathbf{p}}{m_{2}} \approx G m_{4}\left\{\left[\left(\frac{1+\gamma_{2}}{\rho_{4}{ }^{3}}+2 \frac{1+\gamma_{2}^{4}}{r^{3}}\right) \rho_{4} \cos \theta_{4}+\frac{1-\gamma_{2}^{3}}{r^{2}}\right] \hat{\mathbf{a}}_{1}+\left(\frac{1+\gamma_{2}}{\rho_{4}{ }^{3}}-\frac{1+\gamma_{2}^{4}}{r^{3}}\right) \rho_{4} \sin \theta_{4} \hat{\mathbf{a}}_{2}\right\}
$$

The foregoing expressions are evaluated numerically, and the quantities $p_{i}=\left(\mathbf{p} \cdot \hat{\mathbf{a}}_{i}\right) / m_{2}(i=$ $1,2,3)$ and $p=\sqrt{\mathbf{p} \cdot \mathbf{p}} / m_{2}$ are compared in Fig. 6. Solid curves are associated with Eq. (32) and dashed curves represent Eq. (33). The position vector $\mathbf{r}$ is given a magnitude of $1.5015 \times 10^{6} \mathrm{~km}$ and the direction of $\hat{\mathbf{a}}_{1}$. The positions of $P_{3}$ (Sun), $P_{1}$ (Earth), and $P_{4}$ (Moon) are obtained from the ephemerides published in Ref. [12] for a 30-day period beginning on the epoch of March 20, 2000, 16h:40m:00s GMT (Greenwich Mean Time), selected so that Earth lies between the Sun and Moon, and $\boldsymbol{\rho}_{4} \cdot \hat{\mathbf{a}}_{2} \approx 0$. The projection $p_{1}$ along the Sun-Earth line is shown on the upper left in Fig. 6 with a solid curve, and is seen to vary between $3.8 \times 10^{-5} \mathrm{~m} / \mathrm{s}^{2}$ and $-3.4 \times 10^{-5} \mathrm{~m} / \mathrm{s}^{2}$. 

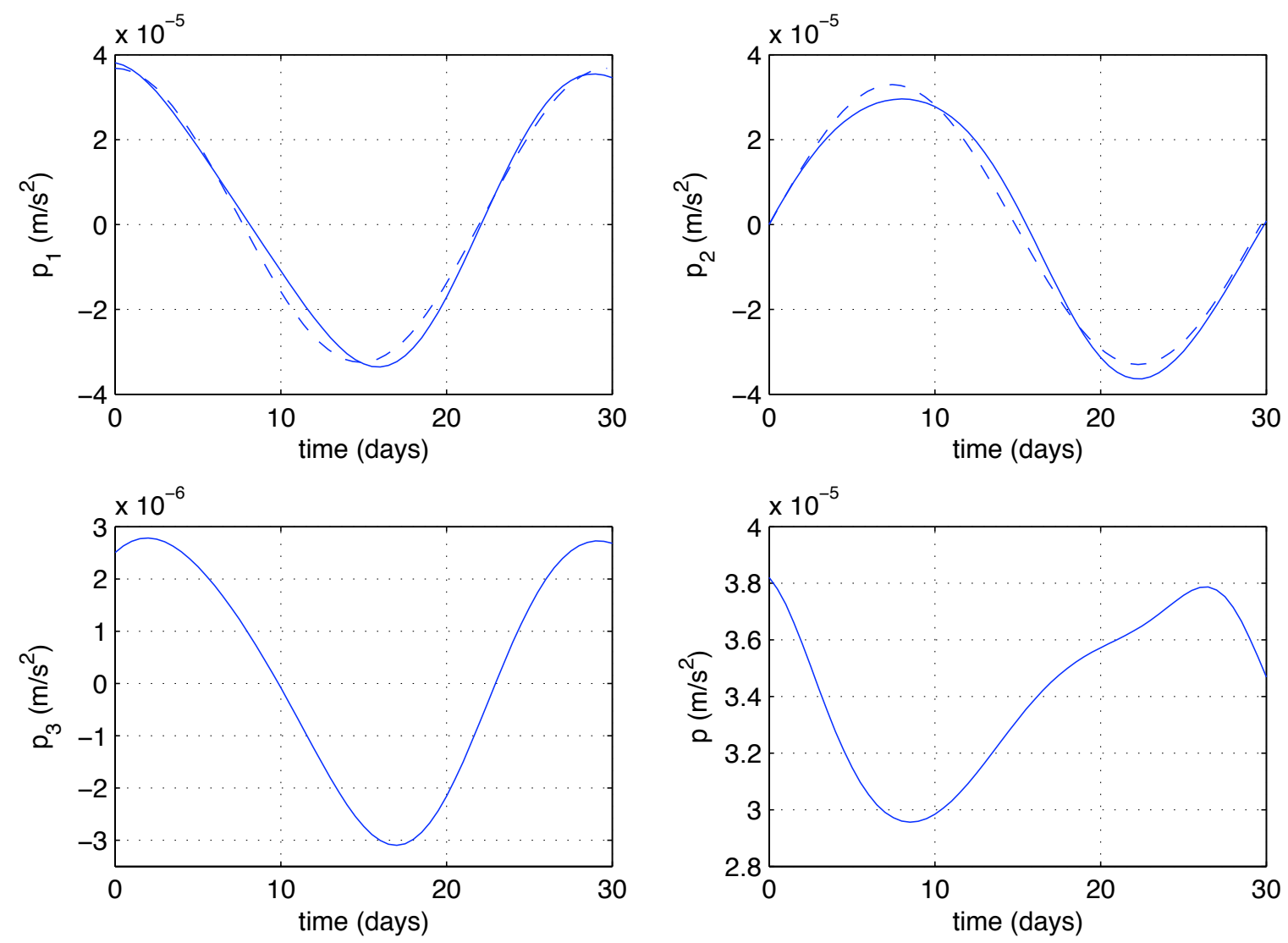

Figure 6: Lunar Perturbing Force Per Unit Mass 
The projection $p_{2}$ in the ecliptic and perpendicular to the Sun-Earth line, displayed in the upper right, varies nearly sinusoidally between $2.9 \times 10^{-5} \mathrm{~m} / \mathrm{s}^{2}$ and $-3.6 \times 10^{-5} \mathrm{~m} / \mathrm{s}^{2}$. The projection $p_{3}$ perpendicular to the Sun-Earth line and to the ecliptic, contained in the lower left, varies nearly sinusoidally between $2.8 \times 10^{-6} \mathrm{~m} / \mathrm{s}^{2}$ and $-3.1 \times 10^{-6} \mathrm{~m} / \mathrm{s}^{2}$, an order of magnitude less than the other two projections. The magnitude $p$ is displayed in the plot on the lower right, and is observed to vary between $3.8 \times 10^{-5}$ and $2.9 \times 10^{-5} \mathrm{~m} / \mathrm{s}^{2}$. The approximate relationships for $p_{1}$ and $p_{2}$ in Eq. (33) are evaluated with the numerical values listed in Table 1 and the lunar synodical period is used to convert the independent variable $\theta_{4}$ to time; the results are depicted with dashed curves in Fig. 6 where it is evident that the approximations are reasonably good for this particular month.

\section{Estimate of $\Delta \mathrm{V}$}

The velocity increments that must be supplied each month by thrusters aimed in the directions of $\hat{\mathbf{a}}_{1}, \hat{\mathbf{a}}_{2}$, and $\hat{\mathbf{a}}_{3}$ are simply the areas under the corresponding three curves of Fig. 6. Approximate relationships formed from Eq. (33) can be integrated analytically to obtain estimates of the velocity increments to be supplied by thrusters parallel to the $\hat{\mathbf{a}}_{1}$ and $\hat{\mathbf{a}}_{2}$ directions. The velocity increment in each direction over one lunar orbit is given by

$$
\Delta V_{i} \triangleq \int_{0}^{2 \pi / n_{s}} \frac{\left|\mathbf{p} \cdot \hat{\mathbf{a}}_{i}\right|}{m_{2}} d t=4 \int_{0}^{\pi / 2} \frac{\left|\mathbf{p} \cdot \hat{\mathbf{a}}_{i}\right|}{n_{s} m_{2}} d \theta_{4} \quad(i=1,2)
$$

where $\theta_{4}=n_{s} t$. The trigonometric functions in Eq. (33) allow setting the upper limit of integration to $\pi / 2$ and quadrupling the result. Consequently,

$$
\begin{aligned}
& \Delta V_{1}=\frac{4 G m_{4}}{n_{s}}\left\{\left[\frac{1+\gamma_{2}}{\rho_{4}^{3}}+\frac{2\left(1+\gamma_{2}^{4}\right)}{r^{3}}\right] \rho_{4}+\frac{\pi}{2} \frac{1-\gamma_{2}^{3}}{r^{2}}\right\} \\
& \Delta V_{2}=\frac{4 G m_{4}}{n_{s}}\left(\frac{1+\gamma_{2}}{\rho_{4}{ }^{3}}-\frac{1+\gamma_{2}^{4}}{r^{3}}\right) \rho_{4}
\end{aligned}
$$

Using the values in Table 1 , one obtains $\Delta V_{1}=62 \mathrm{~m} / \mathrm{s}$ and $\Delta V_{2}=54 \mathrm{~m} / \mathrm{s}$. Hence, the monthly velocity increment needed to keep the spacecraft fixed on the Sun-Earth line is estimated to be $116 \mathrm{~m} / \mathrm{s}$. However, the science requirements of a telescope for observing Earth's atmosphere are satisfied even if it is permitted to take excursions of several thousand km along the Sun-Earth line. In this case the propulsion system need only supply a monthly velocity increment of $54 \mathrm{~m} / \mathrm{s}$, saving more than $50 \%$ of the propellant compared to what must be expended to prevent any lunar-induced excursions. 

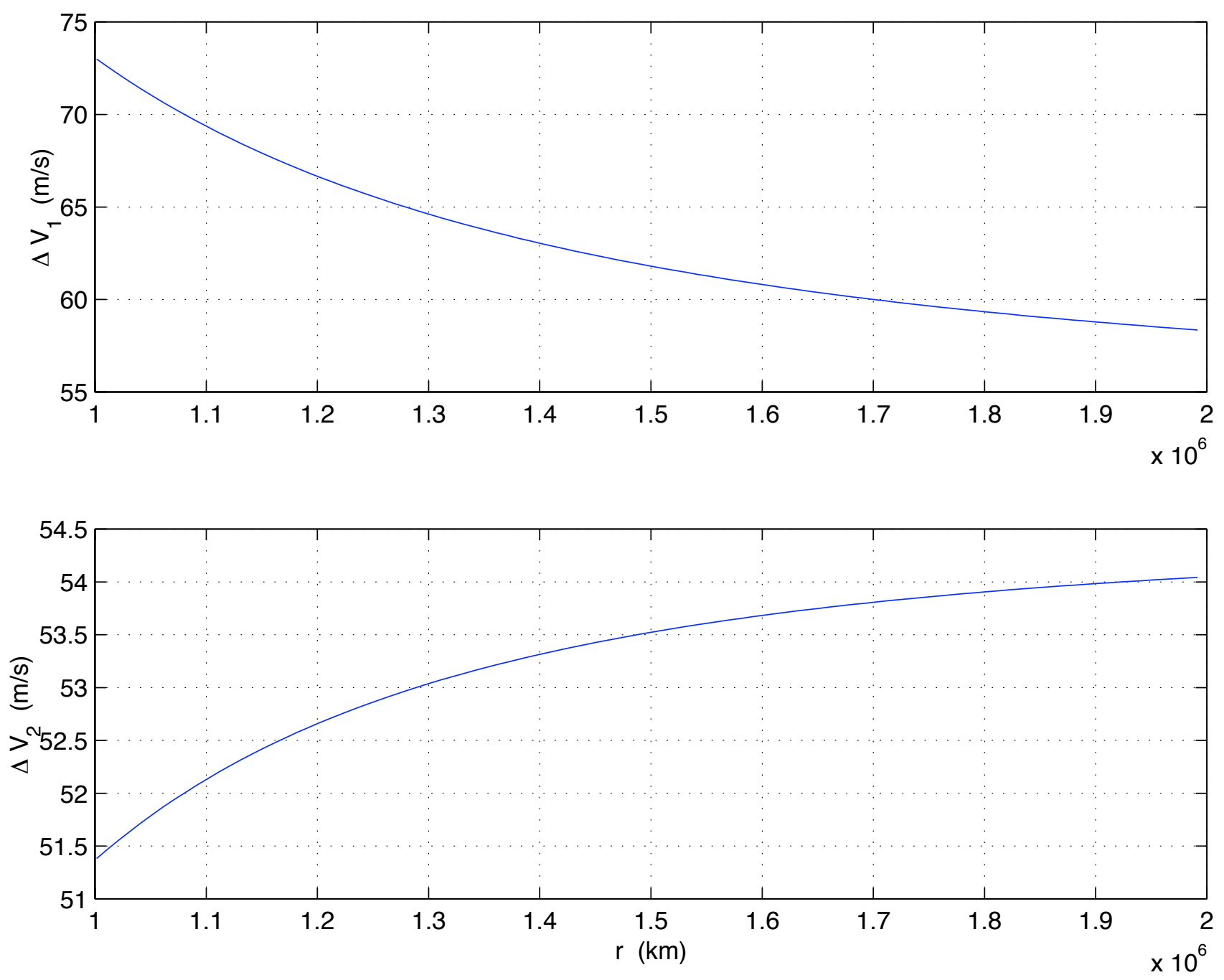

Figure 7: Monthly In-Plane Velocity Increments to Keep Spacecraft Fixed

The monthly values of $\Delta V_{1}$ and $\Delta V_{2}$ needed to keep a spacecraft fixed at other points on the Sun-Earth line are shown in Fig. 7; the range of $r$ considered is within $5 \times 10^{5} \mathrm{~km}$ of the nominal value in Table 1. Variations of the monthly in-plane velocity increments are not drastic, but the cumulative change for a mission lasting several years will of course be significant. The sensitivity of $\Delta V_{2}$ to changes in $r$ is markedly less than that of $\Delta V_{1}$; hence, allowing the spacecraft to move up and down the Sun-Earth line is once again seen to be advantageous.

Keeping a spacecraft fixed at some point on the Sun-Earth line is recognized to be overly restrictive and requires a monthly expenditure of propellant according to the results shown in Fig. 7. On the other hand propellant cost could be virtually eliminated by attempting to keep 

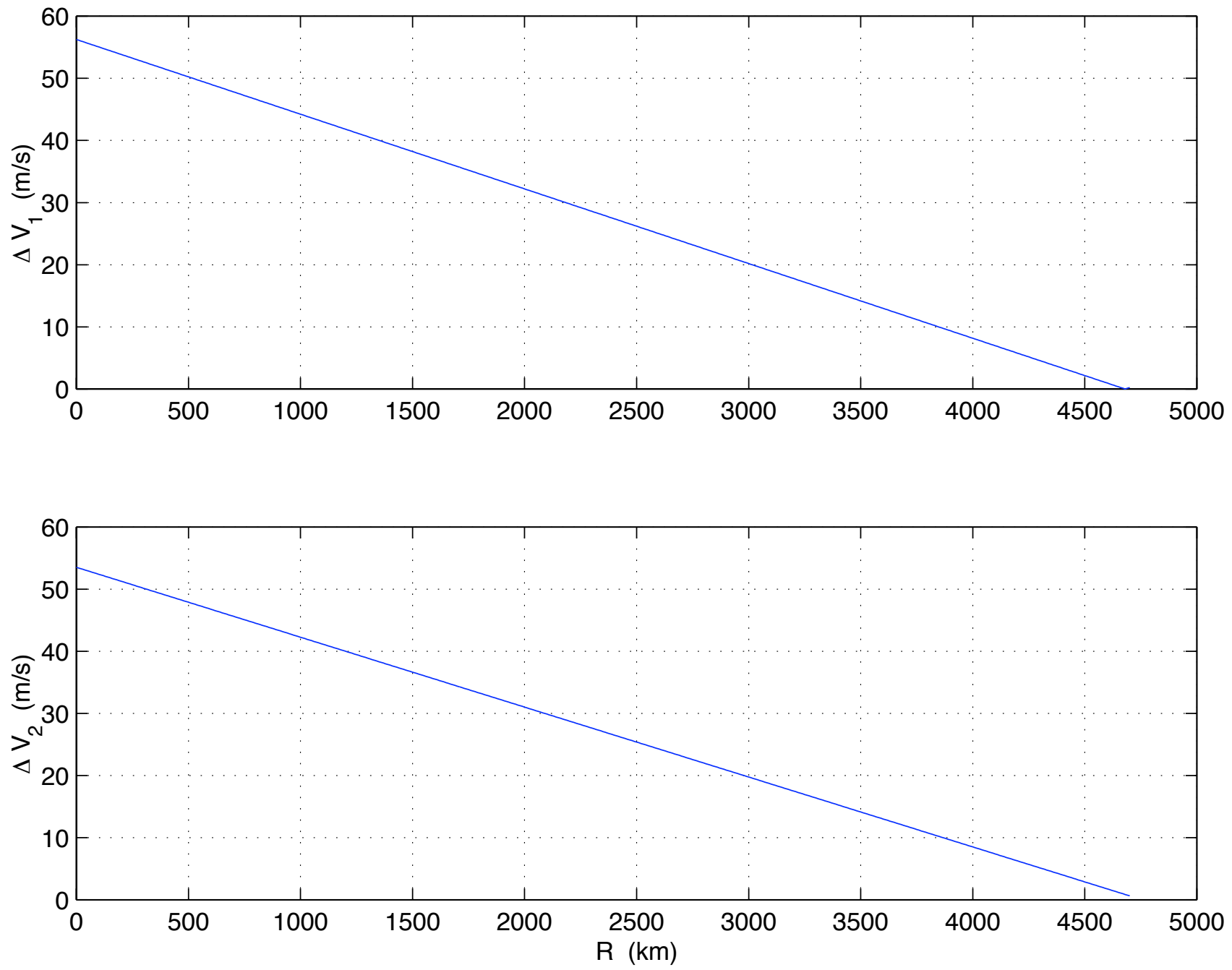

Figure 8: Monthly In-Plane Velocity Increments for Circular Spacecraft Motion 
a spacecraft coincident with the equilibrium point displaced by lunar gravitation as illustrated in Fig. 5, but this is clearly too permissive in view of the science requirements. As has been mentioned already, a compromise between the two extremes of motion is needed. A simple way to investigate the benefits of relaxing the constraint is to prescribe the spacecraft's position over the course of a month and determine the associated velocity increments. For example, motion depicted in Fig. 5 may be regarded as approximately circular and hence be described by the relationships

$$
\begin{aligned}
& x=R \cos n_{s} t+6177 \quad \mathrm{~km} \\
& y=R \sin n_{s} t \quad \mathrm{~km}
\end{aligned}
$$

where the radius $R$ of the circle can be varied. The propulsive force per unit mass needed to bring about the prescribed motion is given by

$$
\begin{aligned}
& p_{1}=\ddot{x}-2 n_{3} \dot{y}-\left(1+2 B_{L}\right) n_{3}{ }^{2} x-f_{1} \\
& p_{2}=\ddot{y}+2 n_{3} \dot{x}+\left(B_{L}-1\right) n_{3}{ }^{2} y-f_{2}
\end{aligned}
$$

where $x, y$, and their first and second derivatives with respect to time are obtained from Eqs. (37) and (38), and $f_{1}$ and $f_{2}$ are given in Eqs. (27) and (28).

In-plane velocity increments are obtained via numerical quadrature in accordance with Eqs. (34) for various radii $R$ and the results are shown in Fig. 8. When $R=0$ the spacecraft is fixed on the Sun-Earth line and $\Delta V_{2}$ has the monthly value of $54 \mathrm{~m} / \mathrm{s}$ as reported previously, whereas $\Delta V_{1}$ is reduced to $56 \mathrm{~m} / \mathrm{s}$ due to the additional displacement of $6177 \mathrm{~km}$ in the direction of $\hat{\mathbf{a}}_{1}$. As expected, $\Delta V_{1}$ and $\Delta V_{2}$ become very small when $R$ reaches $4700 \mathrm{~km}$. The science requirements, however, require $R \leq 200 \mathrm{~km}$.

An elliptical trajectory of appropriate size will allow motion along the Sun-Earth line while constraining the excursion in the perpendicular direction. Equations (37) and (38) can be replaced by

$$
\begin{aligned}
& x=4700 \cos n_{s} t+6177 \quad \mathrm{~km} \\
& y=200 \sin n_{s} t \quad \mathrm{~km}
\end{aligned}
$$

to prescribe motion in a $4700 \times 200 \mathrm{~km}$ ellipse. Numerical quadrature of Eqs. (39) and (40) then yields monthly values of $\Delta V_{1}=6.9 \mathrm{~m} / \mathrm{s}$ and $\Delta V_{2}=44.1 \mathrm{~m} / \mathrm{s}$, for a total of $51 \mathrm{~m} / \mathrm{s}$. 
Reference [13] takes up optimal control of the Earth observation telescope discussed here. An interesting in-plane periodic trajectory in the shape of a candy wrapper is shown to satisfy the contraint $y \leq 200 \mathrm{~km}$, with an associated minimum total monthly $\Delta V$ of less than $40 \mathrm{~m} / \mathrm{s}$. The authors of that work also report a progression of optimal trajectories that take on the shape of a peanut as the constraint is relaxed from $y \leq 0$ until the contraint is absent, whereupon the trajectory becomes similar to what is shown in Fig. 5 .

Mission plans discussed in Ref. [14] call for measurements to be taken with the telescope over a period of 5 to 10 years. The total velocity increment needed for stationkeeping would amount to 3 to $6 \mathrm{~km} / \mathrm{s}$, assuming a conservative monthly figure of $50 \mathrm{~m} / \mathrm{s}$. Mettler et al. note that the mission becomes feasible with the use of a future low-thrust electric propulsion system having a forecast specific impulse of $6000 \mathrm{sec}$. A high-thrust chemical propulsion upper stage would supply $\Delta V=3.2$ $\mathrm{km} / \mathrm{s}$ to depart low Earth orbit and enter a transfer trajectory to $\mathcal{L}_{2}$. The cost of low-thrust orbit insertion near $\mathcal{L}_{2}$ is approximately $336 \mathrm{~m} / \mathrm{s}$. A complete assessment of mission feasibility would require careful study of the cost of orbit determination and maneuver execution errors. ${ }^{4}$

\section{Conclusion}

A spacecraft, stationed on the Sun-Earth line near the trans-Earth Lagrange point $\mathcal{L}_{2}$ in order to obtain measurements of Earth's atmosphere as it occults the Sun, must possess a propulsion system capable of countering the lunar gravitational perturbation. Expressions for the associated force per unit mass and for the corresponding velocity increment needed to keep a vehicle fixed on the Sun-Earth line have been developed and subsequently evaluated numerically. A spacecraft permitted to move along the Sun-Earth line in response to the Moon's influence needs less than half the amount of propellant required to negate completely the lunar disturbance. The benefits of allowing relatively tightly restricted motion in the proximity of $\mathcal{L}_{2}$ are quantified by studying prescribed motion in some simple trajectories.

\footnotetext{
${ }^{4}$ The authors thank Greg Marr, NASA Goddard Space Flight Center, for providing the information regarding the transfer trajectory and orbit insertion.
} 


\section{Acknowledgment}

The research described here was performed as part of the study of an Earth observation telescope at $\mathcal{L}_{2}$, one of the projects in NASA's Revolutionary Aerospace Systems Concepts (RASC) program of 2003. The authors would like to thank Renjith Kumar and Haijun Shen of Analytical Mechanics Associates for a lively exchange of questions and comments related to this work. Our gratitude is extended to two anonymous reviewers for their suggestions for improving the paper. We would also like to thank Darrell Caldwell and Doug Murphy for operating the Synergistic Engineering Environment to produce the views of the Sun occulted by Earth, and Anne Costa for creating the four-body diagrams.

\section{References}

[1] FARQUHAR, R. W., and DUNHAM, D. W., "Use of Libration-point Orbits for Space Observatories," Observatories in Earth Orbit and Beyond, Kluwer Academic Publishers, Dordrecht, Netherlands, 1990, pp. 391-395.

[2] FARQUHAR, R. W., "The Role of the Sun-Earth Collinear Libration Points in Future Space Exploration," Space Times, Nov.-Dec., 2000, pp. 9-12.

[3] WiScombe, W., HERMAN, J., and VAlERO, F., "L-1 and L-2 Observatories for Earth Science in the Post-2010 Era," International Geoscience and Remote Sensing Symposium, 2002, IEEE, IGARSS '02, Vol. 1, 2002, pp. 365-367.

[4] BREAKWELL, J. V., and BROWN, J. V., "The 'Halo' Family of 3-Dimensional Periodic Orbits in the Earth-Moon Restricted 3-Body Problem," Celestial Mechanics, Vol. 20, Nov. 1979, pp. 389-404.

[5] HOWELL, K. C., and BREAKWELL, J. V., "Almost Rectilinear Halo Orbits," Celestial Mechanics, Vol. 32, 1984, pp. 29-52.

[6] FARQUHAR, R. W., "The Moon's Influence on the Location of the Sun-Earth Exterior Libration Point," Celestial Mechanics, Vol. 2, July 1970, pp. 131-133. 
[7] COLOMBO, G., "The Stabilization of an Artificial Satellite at the Inferior Conjunction Point of the Earth-Moon System," Smithsonian Astrophysical Observatory Special Report No. 80, 1961.

[8] NICHOLSON, F. T., "Effect of Solar Perturbation on Motion Near the Collinear Earth-Moon Libration Points," AIAA Journal, Vol. 5, No. 12, Dec. 1967, pp. 2237-2241.

[9] FARQUHAR, R. W., "The Control and Use of Libration-Point Satellites," NASA TR R-346, 1970.

[10] BATTIN, R. H., An Introduction to The Mathematics and Methods of Astrodynamics, AIAA, New York, 1987.

[11] HOWELL, K. C., and GUZMÁN, J. J., "Spacecraft Trajectory Design in the Context of a Coherent Restricted Four-body Problem with Application to the MAP Mission," IAF Paper 00-A.5.06, 51st International Astronautical Congress, Rio de Janeiro, Brazil, Oct. 2-6, 2000.

[12] The Astronomical Almanac for the Year 1999, Nautical Almanac Office, United States Naval Observatory, U.S. Government Printing Office.

[13] SHEN, H., KUMAR, R. R., and SEYWALD, H., "Minimum-Fuel Periodic Orbits in the Vicinity of a Fixed Point on the Sun-Earth Line: The Planar Case," AAS 04-247, Vol. 119, Advances in the Astronautical Sciences, Spaceflight Mechanics 2004, Proceedings of the 14th Annual AAS/AIAA Space Flight Mechanics Meeting, pp. 2263-2276.

[14] METtleR, E., AÇIKMEŞE, A. B., BRECKENRIDGE, W. G., MACENKA, S. A., and TUBBS, E. F., "Earth Atmosphere Observatory Formation at L2," AIAA 2004-5917, Space 2004 Conference and Exhibit, September 28-30, 2004, San Diego, California. 